\title{
SOME RESULTS IN THE FOURIER ANALYSIS
}

\author{
TIKAO TATUZAWA
}

To TADAsI NAKayama in memorial

There are many uses of Fourier analysis in the analytic number theory. In this paper we shall derive two fundamental theorems using Cramer's method (Mathematical methods of statistics, 1946). Let $E, E^{*}$ be unit cubes in the whole $n$-dimensional Euclidean space $X$ such that

$$
\begin{aligned}
& E=\left\{\left(u_{1} \cdots u_{n}\right): 0 \leqq u_{1} \leqq 1, \ldots, 0 \leqq u_{n} \leqq 1\right\} \\
& E^{*}=\left\{\left(u_{1} \cdots u_{n}\right): x_{1}-\frac{1}{2} \leqq u_{1} \leqq x_{1}+\frac{1}{2}, \ldots, x_{n}-\frac{1}{2} \leqq u_{n} \leqq x_{n}+\frac{1}{2}\right\}
\end{aligned}
$$

We define $F(\boldsymbol{u})$ as follows

$$
\begin{gathered}
F(u)=0(u<x-t), \frac{1}{2}(u=x-t), 1(x-t<u<x+t), \\
\frac{1}{2}(u=x+t), 0(x+t<u),
\end{gathered}
$$

for fixed $x$ and $t>0$.

LEMMA 1. For fixed $x$ and $t\left(0<t<\frac{1}{2}\right)$ the function

$$
\sum_{m=-k}^{k} \frac{\operatorname{sim} 2 \pi m(x+t-u)}{2 \pi m}-\sum_{m=-k}^{k} \frac{\sin 2 \pi m(x-t-u)}{2 \pi m}
$$

is boundedly convergent to $F(u)$ as $k \rightarrow \infty$, where $x-\frac{1}{2} \leqq u \leqq x+\frac{1}{2}$.

Proof. Since (1) is equal to

$$
\begin{aligned}
& 2 t+2 \int_{0}^{x+t-u}(\cos 2 \pi z+\cdots+\cos 2 \pi k z) d z-2 \int_{0}^{x-t-u}(\cos 2 \pi z+\cdots+2 \pi k z) d z \\
= & 2 t+2 \int_{x-t-u}^{x+t-u} \frac{\sin \left(k+\frac{1}{2}\right) 2 \pi z-\sin \frac{1}{2} 2 \pi z}{2 \sin \frac{1}{2} 2 \pi z} d z=\int_{x-t-u}^{x+t-u} \frac{\sin (2 k+1) \pi z}{\sin \pi z} d z,
\end{aligned}
$$

the lemma is obtained by proving that

Received March 10, 1965. 


$$
\int_{0}^{a} \frac{\sin (2 k+1) \pi z}{\operatorname{sim} \pi z} d z \quad\left(|a| \leqq t+\frac{1}{2}\right)
$$

is boundedly convergent to $\frac{1}{2}(a>0), 0(a=0),-\frac{1}{2}(a<0)$ as $k \rightarrow \infty$. If we put $2 \pi z=v$, then

$$
\begin{gathered}
\int_{0}^{a} \frac{\sin (2 k+1) \pi z}{\sin \pi z} d z=\frac{1}{z} \int_{0}^{2 \pi a} \frac{\sin \left(k+\frac{1}{2}\right) v}{2 \sin \frac{v}{2}} d v \\
=\frac{1}{\pi}\left\{\int_{0}^{(2 k+1) \pi a} \frac{\sin v}{v} d v+\int_{0}^{2 \pi a}\left(\frac{1}{2 \sin \frac{v}{2}}-\frac{1}{v}\right) \sin \left(k+\frac{1}{2}\right) v d v\right\},
\end{gathered}
$$

whence follows the result by the theorem of Dirichlet's integral.

Lemma 2. Let $f\left(u_{1}, \ldots, u_{n}\right)$ be a function with period 1 for each variable and be L-integrable over $E$. If we write

$$
a\left(m_{1}, \ldots, m_{n}\right)=\int_{E} \cdots \int e^{2 \pi \imath\left(m_{1} u_{1}+\cdots+m_{n} u_{n}\right)} f\left(u_{1} \cdots u_{n}\right) d u_{1} \cdots d u_{n},
$$

then

$$
\sum_{m_{1}=-k}^{k} \cdots \sum_{m_{n}=-k}^{k}\left(\frac{\sin 2 \pi m_{1} t_{1}}{2 \pi m_{1} t_{1}} \cdots \frac{\sin 2 \pi m_{n} t_{n}}{2 \pi m_{n} t_{n}}\right) e^{-2 \pi i\left(m_{1} x_{1}+\cdots+m_{n} x_{n}\right)} a\left(m_{1} \cdots m_{n}\right)
$$

is convergent to

$$
\frac{1}{2 t_{1}} \cdots \frac{1}{2 t_{n}} \int_{x_{1}-t_{1}}^{x_{1}+t_{1}} \cdots \int_{x_{n}-t_{n}}^{x_{n}+t_{n}} f\left(n_{\mathrm{i}} \cdots u_{n}\right) d u_{1} \cdots d u_{n}
$$

as $k \rightarrow \infty$, provided that $0<t_{1}<\frac{1}{2}, \ldots, 0<t_{n}<\frac{1}{2}$.

Proof. By making use of Lebesgue's dominated convergence theorem, it follows from Lemma 1 that

$$
\begin{aligned}
& \int_{E} \cdots \int \prod_{h=1}^{n}\left\{\sum_{m_{h}=-k}^{k} \frac{e^{2 \pi i m_{h}\left(u_{h}-x_{h}\right)} \sin 2 \pi m_{h} t_{h}}{\pi m_{h}}\right\} f\left(u_{1} \cdots u_{n}\right) d u_{1} \cdots d u_{n} \\
= & \int_{E^{*}} \cdots \int \prod_{h=1}^{n}\left\{\sum_{m_{h}=-k}^{k} \frac{\sin 2 \pi m_{h}\left(x_{h}+t_{h}-u_{h}\right)}{2 \pi m_{h}}-\sum_{m_{h}=-t_{k}}^{k} \frac{\sin 2 \pi m_{h}\left(x_{h}-t_{h}-u_{h}\right)}{2 \pi m_{h}}\right\} \\
& f\left(u_{1} \cdots u_{n}\right) d u_{1} \cdots d u_{n} \quad \text { (by translation) } \\
\rightarrow & \int_{x_{1}-t_{1}}^{x_{1}+t_{1}} \cdots \int_{x_{n}-t_{n}}^{x_{n}+t_{n}} f\left(u_{1} \cdots u_{n}\right) d u_{1} \cdots d u_{n} \quad(\text { as } k \rightarrow \infty) .
\end{aligned}
$$

On the other hand, it is easy to verify that $(2)=\frac{1}{2 t_{1}} \cdots \frac{1}{2 t_{n}}(3)$. Hence we 
get the lemma.

THEOREM 1. Let $f\left(u_{1} \cdots u_{n}\right)$ and $a\left(m_{1} \cdots m_{n}\right)$ be the same as in Lemma 2. If

$$
\sum_{m_{1}=-\infty}^{\infty} \cdots \sum_{m_{n}=-\infty}^{\infty}\left|a\left(m_{1} \cdots m_{n}\right)\right|
$$

is convergent, then there exists

$$
\lim _{t_{1} \rightarrow 0, \ldots, t_{n} \rightarrow 0} \frac{1}{2 t_{1}} \cdots \frac{1}{2 t_{n}} \int_{x_{1}-t_{1}}^{x_{1}+t_{1}} \cdots \int_{x_{n}-t_{n}}^{x_{n}+t_{n}} f\left(u_{1} \cdots u_{n}\right) d u_{1} \cdots d u_{n}
$$

and equals

$$
\sum_{m_{1}=-\infty}^{\infty} \cdots \sum_{m_{n}=-\infty}^{\infty} e^{-2 \pi i\left(m_{1} x_{1}+\cdots+m_{n} x_{n l}\right)} a\left(m_{1} \cdots m_{n}\right) .
$$

Proof. Since

$$
\begin{aligned}
& g\left(t_{1} \cdots t_{n}\right) \\
& \quad=\sum_{m_{1}=-\infty}^{\infty} \cdots \sum_{m_{n}=-\infty}^{\infty}\left(\frac{\sin 2 \pi m_{1} t_{1}}{2 \pi m_{1} t_{1}} \cdots \frac{\sin 2 \pi m_{n} t_{n}}{2 \pi m_{n} t_{n}}\right) e^{-2 \pi i\left(m_{1} x_{1}+\cdots+m_{n} x_{n}\right)} a\left(m_{1} \cdots m_{n}\right)
\end{aligned}
$$

is convergent absolutely and uniformly in the neighbourhood of $\left(t_{1} \cdots t_{n}\right)=$ $(0 \cdot 0)$, we have

$$
\left.\lim _{t_{1} \rightarrow 0, \ldots, t_{n \rightarrow 0}} g\left(t_{1} \cdots t_{n}\right)=\sum_{m_{1}=-\infty}^{\infty} \cdots \sum_{m_{n}=-\infty}^{\infty} e^{-2 \pi i i_{m 1} x_{1}+\cdots+m_{n} x_{n}}\right) a\left(m_{1} \cdots m_{n}\right),
$$

whence follows the result by Lemma 2 .

Firstly we note that if $f\left(u_{1} \cdots u_{n}\right)$ is continuous at $\left(x_{1} \cdots x_{n}\right)$, then $(5)$ is equal to $f\left(x_{1} \cdots x_{n}\right)$. Secondly we note that if

$$
\frac{\partial^{p_{1}+\cdots+p_{n}}}{\partial^{p_{1}} x_{1} \cdot \cdots \cdot \partial^{p_{n}} x_{n}} f\left(x_{1} \cdots x_{n}\right)
$$

are continuous in $X$, where $p_{i}(1 \leqq i \leqq n)$ is 0 or 1 , then (4) of Theorem 1 is convergent. This can be obtained by the device of using Bessel's inequality.

TheоRem 2. Let $f\left(u_{1} \cdots u_{n}\right)$ be L-integrable over $X$. We write

$$
g\left(v_{1} \cdots v_{n}\right)=\int_{X} \cdots \int e^{2 \pi i\left(v_{1} u_{1}+\cdots v_{n} u_{n}\right)} f\left(u_{1} \cdots u_{n}\right) d u_{1} \cdots d u_{n}
$$

and assume that $g\left(v_{1} \cdots v_{n}\right)$ is L-integrable over $X$. Then, there exists

$$
\lim _{t_{1} \rightarrow 0, \ldots, t_{n} \rightarrow 0} \frac{1}{2 t_{1}} \cdots \frac{1}{2 t_{n}} \int_{x_{1}-t_{1}}^{x_{1}+t_{1}} \cdots \int_{x_{n}-t_{n}}^{x_{n}+t_{n}} f\left(u_{1} \cdots u_{n}\right) d u_{1} \cdots d u_{n}
$$


Proof. Instead of (1), we use the formula

$$
\begin{gathered}
\lim _{z \rightarrow \infty} \frac{1}{\pi} \int_{-z}^{z} \frac{\sin 2 \pi t v}{v} e^{2 \pi t(u-x) v} d v \\
=\lim _{z \rightarrow \infty}\left\{\int_{-z}^{z} \frac{\sin 2 \pi v(x+t-u)}{2 \pi v} d v-\int_{-z}^{z} \frac{\sin 2 \pi v(x-t-u)}{2 \pi v} d v\right\}=F(u)
\end{gathered}
$$

(boundedly convergent)

By the assumption, the integral

$$
\int_{-z}^{z} \cdots \int_{-z}^{z} \prod_{h=1}^{n}\left\{\frac{\sin 2 \pi t_{h} v_{h}}{2 \pi t_{h} v_{h}} e^{-2 \pi i x_{h} v_{h}}\right\} g\left(v_{1} \cdots v_{n}\right) d v_{1} \cdots d v_{n} .
$$

exists and equals

$$
\frac{1}{2 t_{1}} \cdots \frac{1}{2 t_{n}} \int_{X} \cdots \int_{h=1}^{n}\left\{\frac{1}{\pi} \int_{-z}^{z} \frac{\sin 2 \pi t_{h} v_{h}}{v_{h}} e^{2 \pi i\left(v_{h}-x_{h} ! v_{h}\right.} d v_{h}\right\} f\left(u_{1} \cdots u_{n}\right) d u_{1} \cdots d u_{n}
$$

by Fubini's theorem. Letting $z \rightarrow \infty$ and using the Lebesque dominated convergence theorem, we obtain

$$
\begin{gathered}
\frac{1}{2 t_{1}} \cdots \frac{1}{2 t_{n}} \int_{x_{1}-t_{1}}^{x_{1}+t_{1}} \cdots \int_{x_{n}-t_{n}}^{x_{n}+t_{n}} f\left(u_{1} \cdots u_{n}\right) d u_{1} \cdots d u_{n} \\
=\int_{-\infty}^{\infty} \cdots \int_{-\infty}^{\infty} \prod_{h=1}^{n}\left\{\frac{\sin 2 \pi t_{h} v_{h}}{2 \pi t_{h} v_{h}}\right\} e^{-2 \pi i\left(x_{1} v_{1}+\cdots+x_{n} v_{n}\right)} g\left(v_{1} \cdots v_{n}\right) d v_{1} \cdots d v_{n}
\end{gathered}
$$

by (7). By the same manner as in Theorem 1, we can prove the theorem, and if $f\left(u_{1} \cdots u_{n}\right)$ is continuous at $\left(x_{1} \cdots x_{n}\right)$, then $(6)$ is equal to $f\left(x_{1} \cdots x_{n}\right)$. It should be noted that $g\left(v_{1} \cdots v_{n}\right)$ is always continuous in $X$ by the Lebesque domominated convergence theorem.

Finally we add the Poisson summation formula as an application of Theorem 1. Let $f\left(x_{1} \cdots x_{n}\right)$ be continuous and $L$-integrable over $X$ and

$$
\sum_{k_{1}=-\infty}^{\infty} \cdots \sum_{k_{n}=-\infty}^{\infty} f\left(x_{1}+k_{1} \cdots x_{n}+k_{n}\right)
$$

be uniformly convergent in $E$. We put

$$
a\left(m_{1} \cdots m_{n}\right)=\int_{-\infty}^{\infty} \cdots \int_{-\infty}^{\infty} e^{2 \pi i\left(m_{1} t_{1}+\cdots+m_{n} t_{n}\right)} f\left(t_{1} \cdots t_{n}\right) d t_{1} \cdots d t_{n}
$$

and assume that $\sum_{m_{1}=-\infty}^{\infty} \cdots \sum_{m_{n}=-\infty}^{\infty}\left|a\left(m_{1} \cdots m_{n}\right)\right|$

is convergent. Then 


$$
\sum_{m_{1}=-\infty}^{\infty} \cdots \sum_{m_{n}=-\infty}^{\infty} f\left(m_{1} \cdots m_{n}\right)=\sum_{m_{1}=-\infty}^{\infty} \cdots \sum_{m_{n}=-\infty}^{\infty} a\left(m_{1} \cdots m_{n}\right)
$$

To prove this, let

$$
F\left(x_{1} \cdots x_{n}\right)=\sum_{k_{1}=-\infty}^{\infty} \cdots \sum_{k_{n}=-\infty}^{\infty} f\left(x_{1}+k_{1} \cdots x_{n}+k_{n}\right) .
$$

Since

$$
\begin{aligned}
& =\sum_{k_{1}=-\infty}^{\infty} \cdots \sum_{k_{n}=-\infty}^{\infty} \int_{k_{1}}^{k_{1}+1} \cdots \int_{k_{n}}^{k_{n}+1} e^{2 \pi i\left(m_{1}\left(m_{1}-k_{1}\right)+\cdots+m_{n}\left(t_{n}-k_{n}\right)\right)} f\left(t_{1} \cdots t_{n}\right) d t_{1} \cdots d t_{n} \\
& =\sum_{k_{1}=-\infty}^{\infty} \cdots \sum_{k n=-\infty}^{\infty} \int_{E} \cdots \int e^{2 \pi i\left(m_{1} x_{1}+\cdots+m_{n} x_{n}\right)} f\left(x_{1}+k_{1} \cdots x_{n}+k_{n}\right) d x_{1} \cdots d x_{n} \\
& =\int_{E} \cdots \int e^{2 \pi i\left(m_{1} x_{1}+\cdots+m_{n} x_{n}\right)} F\left(x_{1} \cdots x_{n}\right) d x_{1} \cdots d x_{n},
\end{aligned}
$$

it follows from the assumption and Theorem 1 that

$$
\begin{aligned}
& F\left(x_{1} \cdots x_{n}\right)=\sum_{m_{1}=-\infty}^{\infty} \cdots \sum_{m_{n}=-\infty}^{\infty} a\left(m_{1} \cdots m_{n}\right) e^{-2 \pi i\left(m_{1} x_{1}+\cdots+m_{n} x_{n}\right)} \\
= & \sum_{m_{1}=-\infty}^{\infty} \cdots \sum_{m_{n}=-\infty}^{\infty} \int_{-\infty}^{\infty} \cdots \int_{-\infty}^{\infty} e^{2 \pi i\left(m_{1}\left(t_{1}-x_{1}\right)+\cdots+m_{n}\left(t_{n}-x_{n}\right)\right\}} f\left(t_{1} \cdots t_{n}\right) d t_{1} \cdot \cdots d t_{n} \\
= & \sum_{m_{1}=-\infty}^{\infty} \cdots \sum_{m_{n}=-\infty}^{\infty} \int_{-\infty}^{\infty} \cdots \int_{-\infty}^{\infty} e^{2 \pi i\left(m_{1} u_{1}+\cdots+m_{n} u_{n}\right)} f\left(u_{1}+x_{1} \cdots u_{n}+x_{n}\right) d u_{1} \cdots d u_{n},
\end{aligned}
$$

whence follows the result by putting $x_{1}=\cdots=x_{n}=0$.

Mathematical Institute

Nagoya University 